\title{
Preference for fixed vs. variable amounts of reward'
}

\author{
J. E. R. STADDON AND NAMCY K. IMNIS \\ UNIVERSITY OF TORONTO
}

In a concurrent, two-choice situation pige ons match their relative frequency of responding both to relative frequency and relative amount of reward. In a related situation involving secondary reinforcement, pigeons prefer variable over fixed interval schedules of primary reinforcement. The present experiment found little evidence for a similar preference for variable vs. fixed amounts of reward, suggesting that pigeons integrate amount of reward in a simple, linear fashion.

Pigeons may be trained to peck at either of two response keys. When switching from one key to another is never reinforced ('change-over delay') the relative frequency of pecking on each key may be controlled by a variety of independent variables. One such variable is the relative frequency of reinforcement for pecks on each key. If responding is reinforced on a variableinterval schedule, the relative frequency of responding on each of the keys comes to match the relative frequency of reinforcement (Herrnstein, 1961). The reinforcement in question may either be primary or secondary.

In the latter situation, when pecks at either of two response keys occasionally produce a stimulus in the presence of which further pecks occasionally produce food, pigeons show a preference for variable (as opposed to fixed) interval schedules of primary reinforcement (Herrnstein, 1964). This preference is shown as a higher relative frequency of responding on the key producing the secondary reinforcer associated with the variable-interval schedule of primary reinforcement.

In addition to reinforcement frequency and variability in reinforcement frequency, a third parameter, amount of reinforcement, affects the relative frequency of responding in this situation. If responding on one key produces occasional reinforcements consisting of $3 \mathrm{sec}$. access to grain while responses to the other produce reinforcements consisting of $6 \mathrm{sec}$. access, pigeons tend to respond twice as frequently on the second as the first (Catania, 1963). In this respect, therefore, amount of reward is analogous in its effects to frequency of reward. A natural extension of these results is an experiment to investigate the variable vs. fixed amounts (i.e., durations of access to grain) of reinforcement upon preference in the two-choice situation.

Method

The Ss were two male, White Carneaux pigeons, maintained at $80 \%$ of their free-feeding weights.
The experimental chamber was a two-key version of the conventional Skinner box for pigeons, made by the Grason-Stadler Co. Except for condition VI, the left key was always green and the right red. Effective responses produced an audible 'feedback' click. During the presentation of the reinforcer the house and key lights were turned off and the magazine aperture illuminated. Sessions ended after 60 reinforcements.

Pecks on each key were reinforced according to independently-programmed, identical variable-interval schedules with a mean interreinforcement interval of $1.5 \mathrm{~min}$. The first response on one key following responding on the other started a timer which prevented reinforcement on that key for a period of $1.5 \mathrm{sec}$. Switching from one key to the other was therefore never immediately followed by reinforcement.

After 13 training sessions during which approximately equal responding was established to both keys, the sequence of conditions described in Table 1 occurred.

Condition I was a control condition to see whether identical magazine cycles (reward presentation times) on each key would yield the expected equality of responding. Condition II varied the distribution of magazine cycle times on the right key, keeping the mean of the distribution at $3 \mathrm{sec}$, equal to the magazine cycle on the left key. Condition III still further increased the variance of the distribution of magazine cycle times on the right key. Condition IV varied reward magnitude alone, and conditions V and VI further investigated the effect of variability, controlling for position and/or color preference.

Results

The effects of the conditions are indicated in Fig. 1. The only condition which produced a clearcut effect

Table 1. Order and Duration of Experimental Conditions

\begin{tabular}{|c|c|c|c|}
\hline Condition & $\begin{array}{l}\text { Magazine } \\
\text { Left Key }\end{array}$ & $\begin{array}{l}\text { duration } \\
\text { Right Key }\end{array}$ & Sessions \\
\hline 1 & 3 sec. & 3 sec. & 24 \\
\hline II & 3 sec. & $\begin{array}{l}\text { Mean } 3 \text { sec. } \\
(2,5,4,1,3,4,2,3)\end{array}$ & 7 \\
\hline 111 & 3 sec. & $\begin{array}{l}\text { Mean } 3 \text { sec. } \\
(9,1,1,1)\end{array}$ & 8 \\
\hline IV1 & $2 \mathrm{sec}$. & 4 sec. & 17 \\
\hline V & 3 sec. & $\begin{array}{l}\text { Mean } 3 \text { sec. } \\
(9,1,1,1)\end{array}$ & 40 \\
\hline VI & $\begin{array}{l}\text { Mean } 3 \text { sec. } \\
(9,1,1,1)\end{array}$ & 3 sec. & 16 \\
\hline
\end{tabular}

1. Conditions for bird 437: reversed for bird 435 .

The sequence of successive magazine cycle durations is indicated by the numbers in parentheses. 


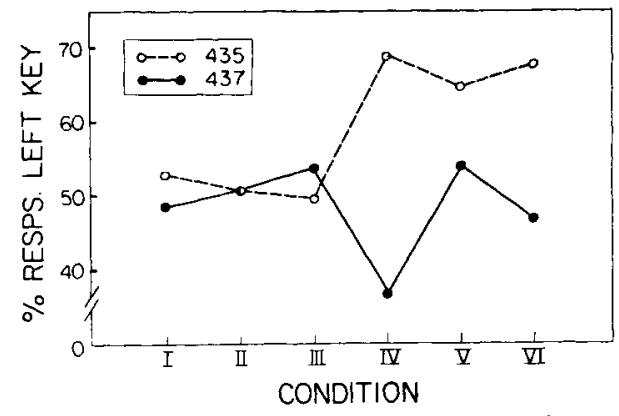

Fig. 1. Mean percent responses on the left key for the last 7 days of each condition.

is condition IV in which the relative frequencies of responding for both pigeons changed in the expected directions by approximately the expected amounts. Thus, bird 435 responded $69 \%$ of the time on the left key (expected: 67\%) while bird 437 responded $37 \%$ (expected: $33 \%$ ) on the left key in approximate agreement with the matching found by Catania (1963).

Conditions II, III, V and VI, the variability conditions, show little effect of the independent variable. For bird 435 conditions II and III show negligible preference for either key, and the left key preference shown in conditions $\mathrm{V}$ and VI seems attributable to the effect of reward magnitude in condition IV. Only the directions of change of preference between conditions are consistently related to the independent variable; thus, between conditions I, II and III the variability of reward duration on the right key increased and preference shifted successively in the direction of that key. Similarly, between conditions V and VI the variable key shifted from right to left and preference also shifted in that direction (the absolute preference in both these conditions was for the left key, however).

Bird 437 shows results of the same general kind, close to indifference between the two keys in conditions II and III and little change between conditions $\mathrm{V}$ and VI. In terms of direction of change, the picture is similar to 435 , but in the opposite direction.

Four conditions in this experiment involved variability in amount of reward. These four conditions allow us to examine three transitions from one level (more or less variability) or position (left variable vs. right variable) of this parameter to another. These transitions are from conditions I to II, II to III and V to VI. Since each bird may prefer either the fixed or the variable key, there are 4 possible outcomes indicating some consistent effect of the independent variable. Since there is a total of 6 transitions for the two birds, yielding 64 possible outcomes (each transition can produce a change in preference towards either fixed or variable keys), the probability of a consistent effect is only $1 / 16$ by chance alone. We cannot, therefore, entirely exclude the possibility of a small effect of variability on each of the two birds.

\section{Discussion}

The concurrent situation, in which Ss may choose to make either of two responses at any time, is a sensitive measure of preference, provided switching from one response to the other is never reinforced (Herrnstein, 1961). Using this procedure with two pigeons we found no substantial effect of variability in reward presentation time on preference. The effectiveness of the situation was demonstrated by a condition in which the mean reward presentation time differed on the two keys. The birds adapted to this change by shifting to the key giving longer access to the reward, in approximate agreement with the matching found by Catania (1963). This condition showed that these pigeons were sensitive to the reward-presentation-time dimension. The next two conditions ( $V$ \& VI), which were replications of the previous variability condition, still failed to show substantial effects of the independent variable; although further analysis indicates that variability may exert a small, idiosyncratic effect.

Herrnstein (1964) found that pigeons show a preference for variable as opposed to fixed interval schedules of reinforcement. One implication of this result is that the function relating frequency of reinforcement to rate of responding is not linear: response rate is controlled by some other measure of reinforcement frequency than the arithmetic mean under these conditions.

The results presented here suggest that the opposite is true of reward magnitude; over the range investigated pigeons appear to integrate reward presentation time in a simple linear fashion.

\section{References}

Catania, A. C. Concurrent Performances: A baseline for the study of reinforcement magnitude. J. exp. Anal. Behav., 1963, 6, 299300 .

Hermstein, R. J. Relative and absolute strength of response as a function of frequency of reiaforcement. J. exp. Anal. Behav., $1961,4,267-272$.

Herrnstein, R. J. Aperiodicity as a factor in choice. J. exp, Anal. Behav., 1964, 7, 179-182.

\section{Note}

1. Supported by the University of Toronto and by National Research Council of Canada Grant APA-155. 